\title{
Oxygen Enrichment Ameliorates Cardiorespiratory Alterations Induced by Chronic High-Altitude Hypoxia in Rats
}

\author{
Xi Shao ${ }^{1 t}$, Xu Dong ${ }^{2 t}$, Jing Cai ${ }^{1,3 t}$, Chi Tang ${ }^{1}$, Kangning Xie ${ }^{1}$, Zedong Yan ${ }^{1}$, Erping Luo ${ }^{1 *}$ \\ and Da Jing ${ }^{1 *}$
}

${ }^{1}$ Department of Biomedical Engineering, Fourth Military Medical University, Xi'an, China, ${ }^{2}$ Recuperation Management Office, Department of Medical Management and Training, Qingdao Special Service Recuperation Center of PLA Navy, Qingdao,

China, ${ }^{3}$ College of Basic Medicine, Shaanxi University of Chinese Medicine, Xianyang, China

OPEN ACCESS

Edited by:

Annalisa Cogo,

University of Ferrara, Italy

Reviewed by:

Jorge G. Farias,

University of La Frontera, Chile

Tadej Debevec,

University of Ljubljana, Slovenia

*Correspondence:

Erping Luo

luoerping@fmmu.edu.cn

Da Jing

jingdaasq@126.com

tThese authors have contributed equally to this work and share first

authorship

Specialty section:

This article was submitted to Environmental, Aviation and Space

Physiology,

a section of the journal

Frontiers in Physiology

Received: 11 October 2020

Accepted: 07 December 2020

Published: 07 January 2021

Citation:

Shao X, Dong X, Cai J, Tang C, Xie K, Yan Z, Luo E and Jing D (2021)

Oxygen Enrichment Ameliorates Cardiorespiratory Alterations Induced

by Chronic High-Altitude Hypoxia in Rats.

Front. Physiol. 11:616145.

doi: 10.3389/fphys.2020.616145
Chronic high-altitude hypoxia $(\mathrm{HAH})$ results in compensatory pathological adaptations, especially in the cardiorespiratory system. The oxygen enrichment technology can provide long-lasting oxygen supply and minimize oxygen toxicity, which has proven to be effective to increase oxygen saturation, decrease heart rate, and improve human exercise performance after ascending to high altitudes. Nevertheless, it remains unknown whether oxygen enrichment can resist chronic HAH-induced cardiorespiratory alterations. Thirty-six male rats were equally assigned to the normal control (NC), $\mathrm{HAH}$, and $\mathrm{HAH}$ with oxygen enrichment $(\mathrm{HAHO}$ ) groups. The $\mathrm{HAH}$ and $\mathrm{HAHO}$ rats were housed in a hypobaric hypoxia chamber equivalent to 5,000 $\mathrm{m}$ for 4 weeks. The HAHO rats were exposed to oxygenenriched air for $8 \mathrm{~h} /$ day. We found that oxygen enrichment mitigated the augmented skin blood flow and improved the locomotor activity of $\mathrm{HAH}$-exposed rats. Oxygen enrichment inhibited $\mathrm{HAH}$-induced increase in the production of red blood cells (RBCs). The hemodynamic results showed that oxygen enrichment decreased right ventricular systolic pressure (RVSP) and mean pulmonary artery pressure (mPAP) in HAH-exposed rats. $\mathrm{HAH}$-associated right ventricular hypertrophy and cardiomyocyte enlargement were ameliorated by oxygen enrichment. Oxygen enrichment inhibited $\mathrm{HAH}$-induced excessive expression of cytokines associated with cardiac hypertrophy and myocardial fibrosis [angiotensin-converting enzyme (ACE)/angiotensin-converting enzyme 2 (ACE2), angiotensin II (Ang II), collagen type I alpha 1 (Col1 $\alpha 1)$, collagen type III alpha 1 (Col3 $\alpha 1)$, and hydroxyproline] in the right ventricle (RV). Oxygen enrichment inhibited medial thickening, stenosis and fibrosis of pulmonary arterioles, and cytokine expression related with fibrosis (Col1 $\alpha 1$, Col3 $\alpha 1$, and hydroxyproline) and pulmonary vasoconstriction [endothelin-1(ET-1)] in HAH-exposed rats. This study represents the first effort testing the efficacy of the oxygen enrichment technique on cardiopulmonary structure and function in chronic HAH animals, and we found oxygen enrichment has the capability of ameliorating chronic HAH-induced cardiopulmonary alterations.

Keywords: oxygen enrichment, cardiorespiratory system, high-altitude hypoxia, chronic mountain sickness, pulmonary hypertension 


\section{INTRODUCTION}

High altitude is defined as an elevation greater than $2,500 \mathrm{~m}$ above sea level with decreased barometric pressure and air density (Hackett and Roach, 2001). Although the proportion of oxygen remains constant, inspired partial pressure of oxygen decreases progressively with the falling barometric pressure at high altitudes (West, 2012). The reduced ambient oxygen pressure induces decrease in the arterial partial pressure of oxygen and arterial oxygen saturation, leading to subsequent oxygen deprivation of tissues, which is known as high-altitude hypoxia (HAH; Murray et al., 2018). Over 140 million people live at high-altitude regions exceeding 2,500 m, and numerous lowlanders commute to high altitudes for work or entertainment (Moore, 2001; West, 2015). Migrants ascending to high altitudes often suffer from acute mountain sickness with symptoms, like headache, insomnia, and fatigue (Hackett and Roach, 2001), while native or longtime dwellers at high altitudes have a high risk of developing chronic mountain sickness due to compensatory adaptation of organism to chronic $\mathrm{HAH}$, and the risk is greater as the altitude increases (Azad et al., 2017). Exaggerated polycythemia represent the key characteristic of acclimation to chronic $\mathrm{HAH}$, which is associated with increased blood viscosity and blood volume (León-Velarde and Richalet, 2006; Tremblay et al., 2019). Long-term HAH exposure also results in pulmonary hypertension with increased pulmonary vascular resistance due to pulmonary vasoconstriction and vascular remodeling (Penaloza and Arias-Stella, 2007). Pulmonary hypertension increases right ventricular afterload, and thus results in compensatory cardiac structural changes, characterized by right ventricular hypertrophy (Vonk Noordegraaf et al., 2017). Individuals exposed to chronic $\mathrm{HAH}$ also suffer from deteriorated cardiac function, such as increased heart rate, decreased stroke volume, and maximal cardiac output (Boussuges et al., 2000; León-Velarde et al., 2010). Chronic HAH-induced cardiorespiratory abnormalities are usually irreversible, which greatly reduces the quality of life and shortens life expectancy of highlanders (Vargas and Spielvogel, 2006; Yang et al., 2015). Therefore, it is of great significance to develop effective prevention strategies to protect cardiopulmonary function and reduce the risk of chronic $\mathrm{HAH}$-associated cardiorespiratory diseases for native and long-term residents at high altitudes.

Several techniques have been reported to mitigate the detrimental effects induced by $\mathrm{HAH}$, such as hyperbaric oxygen chamber, portable hyperbaric chamber, bottled oxygen, and oxygen concentrator based on molecular sieve. It has been demonstrated that hyperbaric oxygen chamber has the capability of increasing arterial oxygen saturation and attenuating HAH-related sickness (Kasic et al., 1991; Zhu et al., 2015). Nonetheless, the inflexibility and potential risks (e.g., middle ear barotrauma, temporary myopia, and pulmonary dyspnea) restrict its widespread application at high altitudes (Heyboer et al., 2017). The portable hyperbaric chamber has been proven effective to improve the symptoms of $\mathrm{HAH}$, whereas the longduration stays in the chamber may lead to issues like vomiting and claustrophobia (Bärtsch, 1992; Davis and Hackett, 2017). Although bottled oxygen (oxygen gas or liquid oxygen) can offer high-concentration oxygen to ameliorate $\mathrm{HAH}$-induced adverse effects (Peacock, 1998), this approach has some negligible limitations, such as the potential oxygen poisoning and inability of long-run supply (Deneke and Fanburg, 1980). Oxygen concentrator based on molecular sieve can remove the nitrogen from air to obtain high-concentration oxygen (80-95\%), which has been widely used for long-term oxygen therapy (Bolton et al., 2006; Nasilowski et al., 2008). However, the low flow rate and potential oxygen toxicity may be its major limitations (Adde et al., 2013). A growing body of studies has raised the interest in the technology of oxygen enrichment, which has proven to be an effective strategy to decrease the equivalent altitude (every $1 \%$ increase in oxygen concentration results in the reduction in equivalent altitude by about $300 \mathrm{~m}$; West, 2002, 2003, 2012). This technique can not only provide long-lasting supply of oxygen enriched air with high flow rate, but also minimize the potential oxidative stress injury (Nagatomo et al., 2012). Recently, our group has developed a series of new oxygen enrichment instruments (including the manpack version, vehiclemounted version, and room-mounted version) based on the membrane gas separation technique (Shen et al., 2013). Studies from our and other groups have shown that the oxygen enrichment technique has the capability of improving the oxygen saturation and decreasing the heart rate of individuals exposed to $\mathrm{HAH}$ (Mcelroy et al., 2000; Shen et al., 2013; Moraga et al., 2018). However, little is known about the efficacy of oxygen enrichment for chronic mountain sickness, especially for chronic HAH-induced damage to the cardiopulmonary system either experimentally or clinically. Therefore, it is necessary to identify the therapeutic effects of the oxygen enrichment technique on chronic $\mathrm{HAH}$-induced cardiorespiratory dysfunction in animals, which can provide rationale for future clinical trials as well as reasonable and scientific application for individuals at high altitudes.

In this study, the chronic HAH rat model was established in a hypobaric hypoxia chamber and then exposed to oxygenenriched air using on our oxygen enrichment device. Then, the effects of oxygen enrichment on the cardiovascular and pulmonary systems in chronic $\mathrm{HAH}$ rats were evaluated via systemic behavioral, hematological, histomorphological, and molecular assessments. The present study represents the first effort to characterize the potential effects of oxygen enrichment on chronic $\mathrm{HAH}$-induced alterations in cardiorespiratory structure and function in animals.

\section{MATERIALS AND METHODS}

\section{Animals and Experimental Design}

Thirty-six male Sprague-Dawley rats (7-8 weeks old, weighing $260-320 \mathrm{~g}$ ) used in the present study were obtained from the Animal Center of the Fourth Military Medical University. Rats were housed under controlled temperature $\left(23 \pm 1^{\circ} \mathrm{C}\right)$ and humidity (40-60\%) with a $12 \mathrm{~h}$ light/dark cycle. Animal experiments were performed in accordance with the Guide for the Care and Use of Laboratory Animals published by the National Institutes of Health. Animals were randomly assigned into the following groups: the normal control group (NC, $n=12)$, the HAH group $(n=12)$, and the HAH with oxygen 
enrichment group (HAHO, $n=12$ ). Rats in the NC group were housed under normoxic conditions at an altitude of $400 \mathrm{~m}$ in the city of Xian. Rats in the HAH group and HAHO group were housed in the hypobaric hypoxia chamber with $22 \mathrm{~h}$ /day for 4 consecutive weeks, while rats in the HAHO group were subjected to oxygen enrichment treatment $(8 \mathrm{~h} /$ day) using the oxygen enrichment device developed by our group for 4 weeks. The hypobaric chamber was kept open for $2 \mathrm{~h}$ per day to replenish food and water, change bedding, clean the chamber and cage, and set up the hypobaric chamber (Titus et al., 2007). The body weight, food intake, and water intake of rats in each group were measured weekly during the experiment. The overall experimental protocol of this study is illustrated in Figure 1.

\section{Hypobaric Hypoxia Exposure and Oxygen Enrichment}

A commercial hypobaric hypoxia chamber (\#LAT-SY01, Suzhou Liante Medical Equipment Co., Ltd., Suzhou, China) was used with the chamber pressure maintained at $54 \mathrm{kPa}$ and oxygen concentration at $20.9 \%$ to simulate the barometric pressure equivalent to an altitude of 5,000 m. A vacuum pump was used to evacuate the air out of the chamber. Solenoid valves were used to precisely control the proportion of gas into or out of the chamber to ensure adequate air circulation. The ascent and descent rates of the hypobaric hypoxia chamber were $91.3 \mathrm{~Pa} / \mathrm{s}$. Local oxygen-enriched environment was established in the hypobaric hypoxia chamber with the combination of a portable oxygen enrichment device and individually ventilated cages (IVC) for rats (Figure 1). The novel oxygen enrichment device was developed by our group based on the oxygen enrichment membrane technique (China Patent No. ZL201210309573.3 and ZL201310468909.5), which has been described in detail in our previous study (Shen et al., 2013). In brief, the device consisted of a ventilation fan, a vacuum pump, a gas buffer, and 16 parallelized oxygen enrichment membrane units. The surface of the oxygen enrichment membrane was coated with a selective layer of polymer solution for high oxygen permeance. Since oxygen permeates through the membrane faster than nitrogen, the membrane exhibits high oxygen/nitrogen selectivity. The vacuum controller was utilized to monitor the pressure of the oxygen-enriched air and suck the air out from membranes. The equipment can produce oxygen-enriched gas with steady oxygen concentration and flow rate both in the hypoxia chamber and in Tibet of China (Shen et al., 2013). The oxygen concentration of the enriched air was measured using an oxygen analyzer (OXYMAT 61, Siemens, Erlangen, Germany). According to the measurements, the oxygen concentration of the air generated by the device was maintained in the range of $28.2-29.3 \%$ at the simulated altitude from 3,000 to $5,000 \mathrm{~m}$ (Figure 1).

\section{Skin Perfusion Measurements}

A Laser Doppler flowmetry (LDF, Moor Instruments Ltd., Axminster, United Kingdom) was utilized to measure the skin microvascular blood flux for all rats in each group following the manufacturer's guidelines. Rats were anesthetized intraperitoneally using sodium pentobarbital $(30 \mathrm{mg} / \mathrm{kg}$ body weight). After calibration, the laser probe (MP7-V2) was positioned on the plantar surface of the left hind paws with a laser wavelength of $780 \mathrm{~nm}$. Rats were placed in the prone position and the probe was held for a period of $1 \mathrm{~min}$.
A

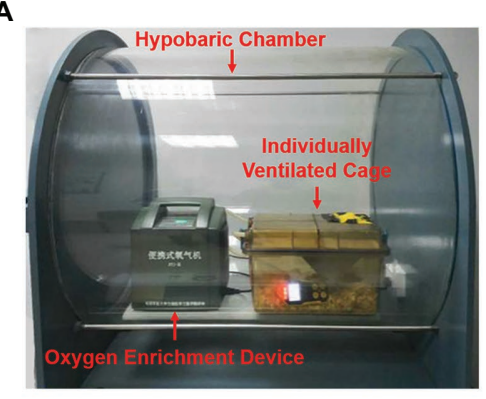

B

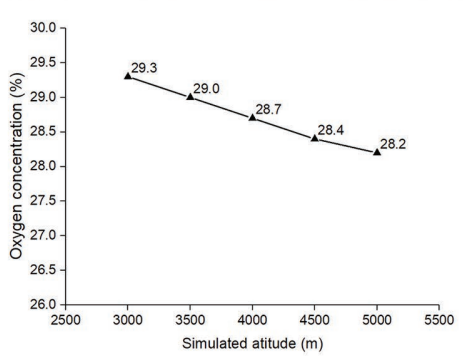

C

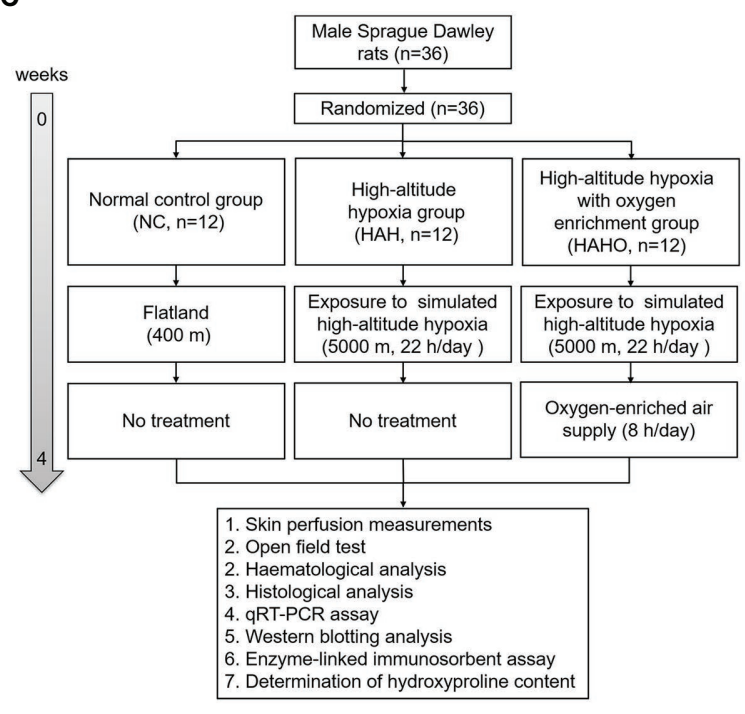

FIGURE 1 | Experimental setup and experimental protocol employed in the present study. (A) Local oxygen-enriched environment was constructed in the hypobaric hypoxia chamber with the combination of the portable oxygen enrichment device and individually ventilated cages (IVCs) for rats. (B) The oxygen concentration in the gas generated by the oxygen enrichment device at various simulated altitudes $(3,000-5,000 \mathrm{~m})$. (C) The flow diagram of the experimental protocol used in this study. 
The cutoff frequency of the low-pass filter was set at $22.5 \mathrm{kHz}$ and the time constant was defined as $0.1 \mathrm{~s}$ to minimize the interference. Data were recorded continuously at the sampling rate of $40 \mathrm{~Hz}$ and transferred to the laptop for further analysis based on the software provided by the manufacturer (moorVMS-PC software). Mean skin blood flux was measured and expressed in arbitrary units (AUs).

\section{Open Field Testing}

Spontaneous locomotor activities of all rats in each group were assessed using the activity cage apparatus (Model 7,420, Ugo Basile, Verase, Italy), which comprised an electronic unit and two sets of emitter/sensor arrays to transmit and detect the invisible infrared beams. Rats were placed in the center of the cage and allowed to move freely. After the rats were acclimated to the apparatus for $10 \mathrm{~min}$, the infrared beam interruptions caused by the movements of rats were automatically collected by the electronic unit for a period of $10 \mathrm{~min}$. Data were analyzed for the measurements of the horizontal activity (crossed beams) and vertical activity (crossed beams).

\section{Hemodynamic Measurements}

Hemodynamics of all rats in each group were measured by the right heart catheterization (RHC). After anesthesia with sodium pentobarbital, rats were stabilized on the operating table. An incision was created on the skin from the mandible to sternum. After surrounding tissues were gently dissected, the right external jugular vein was isolated and ligated proximally with a loose knot. Then, a small hole was created distally to the ligature. A catheter was inserted into the hole of the vein, and then the knot was tightened. The catheter, connected to a hemodynamic monitor (Hewlett-Packard, Palo Alto, CA, United States), was gently moved into the right ventricle (RV) and pulmonary artery to measure the right ventricular systolic pressure (RVSP, $\mathrm{mmHg}$ ) and mean pulmonary artery pressure (mPAP, $\mathrm{mmHg}$ ), respectively.

\section{Hematological Analysis}

After the RHC measurement, cardiac puncture of all rats in each group was performed to collect blood from left ventricle (LV) using a $5 \mathrm{ml}$ sterile syringe. Blood samples were transferred into sample bottles containing ethylenediaminetetraacetate (EDTA) and immediately analyzed by an automatic hematology analyzer (XN-3000, Sysmex, Kobe, Japan). Hematological parameters were determined as follows: red blood cell (RBC, $\left.10^{12} / \mathrm{L}\right)$, hemoglobin $(\mathrm{Hb}, \mathrm{g} / \mathrm{L})$, hematocrit (Hct, \%), mean corpuscular volume $(\mathrm{MCV}, \mathrm{fl})$, mean corpuscular hemoglobin $(\mathrm{MCH}, \mathrm{pg})$, and white blood cell (WBC, $\left.10^{9} / \mathrm{L}\right)$.

\section{Determination of Ventricular Weight}

After cardiac puncture, heart tissues of all rats in each group were isolated and washed repeatedly with ice-cold phosphatebuffered saline (PBS) to remove the remaining blood. After removal of atria, pulmonary trunk, and vessels from the ventricles, the RV was isolated from the LV and ventricular septum. After completely drying PBS with filter papers, weights of the RV free wall, LV free wall, and ventricular septum were determined by an electronic balance (Tianchen instrument, Beijing, China). The heart weight (HW), left ventricular weight (LVW), and right ventricular weight (RVW) were measured. The RV weight to $L V$ plus septum weight ratio $[\mathrm{RV} /(\mathrm{LV}+\mathrm{S})$; Fulton index) was calculated as an index of RV hypertrophy.

\section{Histological Analysis}

After sacrifice, cardiac muscle tissues from six rats in each group were used for histological analysis, and cardiac muscle tissues of the remaining six rats in each group were used for molecular expression assays. The right lung tissues from all rats in each group $(n=12)$ were used for histological analysis, and the left lung tissues were used for molecular expression assays. The isolated heart and right upper lobe tissues were fixed in $10 \%$ formalin overnight. Then, specimens were dehydrated with a graded series of ethanol, embedded in paraffin, and cut into $5-\mu \mathrm{m}$ slices using a diamond saw microtome (Leica 2500E, Leica SpA, Milan, Italy). Specimens of the cardiac muscle tissues were stained with $\mathrm{H} \& \mathrm{E}$ and imaged under an optical microscope (Olympus, Tokyo, Japan) at $400 \times$ magnification to assess the myocardial morphology. The crosssectional area $\left(\mu \mathrm{m}^{2}\right)$ of the cardiomyocyte was determined using ImageJ (National Institutes of Health, Bethesda, Maryland, United States). For lung tissues, sections were stained with $\mathrm{H} \& \mathrm{E}$ and Van Gieson (VG) to observe the morphology and collagen deposition of small pulmonary arteries, respectively. Small pulmonary arteries (50-100 $\mu \mathrm{m}$ in diameter) were randomly selected to measure internal diameter (ID), external diameter (ED), vascular lumen area (VA), and total vascular area (TA). Then, the percentage of medial wall thickness (MWT\%) was calculated as $[\mathrm{MWT} \%=(\mathrm{ED}-\mathrm{ID}) / \mathrm{ED} \times 100 \%]$. The percentage of vascular wall area (WA\%) was calculated as $[\mathrm{WA} \%=(\mathrm{TA}-$ $\mathrm{VA}) / \mathrm{TA} \times 100 \%]$.

\section{Real-Time PCR}

Total RNA was extracted from heart or lung tissues using TRIzol (Invitrogen, Carlsbad, CA, United States). The concentration and purity of RNA were confirmed by the UV spectrophotometer (Bio-Rad, Hercules, CA, United States). Total RNA was synthesized for cDNA using the PrimeScript ${ }^{\mathrm{TM}}$ RT Master Mix (Takara, Ohtsu, Japan) according to the manufacturer's guidelines. The primers of hypoxia-inducible factor-1 alpha (HIF-1 $\alpha$ ), angiotensin-converting enzyme (ACE), angiotensin-converting enzyme 2 (ACE2), collagen type I alpha 1 (Col1 $\alpha 1)$, collagen type III alpha $1(\mathrm{Col} 3 \alpha 1)$, and GAPDH were designed using Primer Premier 5.0 (PREMIER Biosoft International, Palo Alto, CA, United States) and synthesized by Takara Biotechnology Co. Ltd. (Table 1). The RCR mix consisted of $10 \mu \mathrm{l}$ SYBR Premix Ex Taq ${ }^{\mathrm{TM}}$ II (Takara), $1.6 \mu \mathrm{l}$ cDNA, $1.6 \mu \mathrm{l}$ of the upstream and downstream primers, and

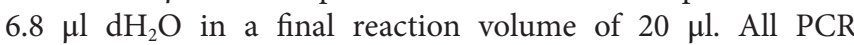
reactions were performed in the CFX96 Real-Time PCR Detection System (Bio-Rad) under the following conditions: initial denaturation at $95^{\circ} \mathrm{C}$ for $3 \mathrm{~min}$, and followed by 40 cycles consisting of denaturation at $95^{\circ} \mathrm{C}$ for $30 \mathrm{~s}$, annealing at $60^{\circ} \mathrm{C}$ 
TABLE 1 | The sequence of primers used in the present study for real-time PCR.

\begin{tabular}{lll}
\hline Genes & Primers & Primer sequence (5'-3') \\
\hline HIF-1 $\alpha$ & Forward & CCAGATTCAAGATCAGCCAGCA \\
& Reverse & GCTGTCCACATCAAAGCAGTACTCA \\
ACE & Forward & TGCCTCAGCCTGGGACTTCTA \\
& Reverse & CCCATTCGTGGTGGGCTA \\
ACE2 & Forward & GGTCTTCTGCCATCCAATITC \\
& Reverse & ACCATCCACCTCCACTTCTCTAAC \\
Col1 $\alpha 1$ & Forward & GACATGTCAGCTTGTGGACCTC \\
& Reverse & AGGGACCCTTAGCCATTGTGTA \\
Col3 $\alpha 1$ & Forward & TTGGCACAGCAGTCCAATGTA \\
& Reverse & GACAGATCCCGAGTCGCAGA \\
GAPDH & Forward & GGCACAGTCAAGGCTGAGATG \\
& Reverse & ATGGTGGTGAGACGCCAGTA \\
\hline
\end{tabular}

for $30 \mathrm{~s}$, and extension at $65^{\circ} \mathrm{C}$ for $10 \mathrm{~s}$. The target gene expression was normalized to GAPDH using the $2^{-\Delta \Delta \mathrm{Ct}}$ method.

\section{Western Blotting}

Total protein was extracted from cardiac muscle tissues using RIPA buffer supplemented with PMSF. The protein concentration was quantified using a BCA protein assay kit (DingguoChangsheng Biotech Co., Beijing, China). The protein was separated in $6 \%$ or $10 \%$ SDS-PAGE and transferred to the NC membrane (Millipore Corp., Bedford, Mass, United States) by electroblotting. Then, membranes were blocked in 5\% skim milk for $1 \mathrm{~h}$ at room temperature and incubated at $4^{\circ} \mathrm{C}$ overnight with the primary antibodies of HIF- $1 \alpha$, ACE, ACE2, and GAPDH (Bioworld, Atlanta, GA, United States). After washing with Trisbuffered saline containing $0.5 \%$ Tween-20 (TBST), membranes were incubated with the secondary antibody for $2 \mathrm{~h}$ at room temperature. The blots were developed using the SuperSignal West Pico chemiluminescent substrate kit (Thermo Scientific, Rockford, IL, United States) and visualized by an ECL chemiluminescent system (GE ImageQuant 350, GE Healthcare).

\section{Enzyme-Linked Immunosorbent Assay}

The protein levels of angiotensin II (Ang II) in myocardial tissues and endothelin-1 (ET-1) in lung tissues were evaluated using the ELISA kits (Nanjing Jiancheng Bioengineering Institute, Nanjing, China). Samples (100 mg) were homogenized in $900 \mu \mathrm{l}$ of $1 \times$ PBS ( $\mathrm{pH}: 7.0-7.4)$ at $4^{\circ} \mathrm{C}$. The homogenates were centrifuged at $12,000 \mathrm{r} / \mathrm{min}$ for $10 \mathrm{~min}$, and the supernatant was collected for the measurements. All procedures of the ELISA assays were strictly performed according to the manufacturer's protocol.

\section{Determination of Hydroxyproline Content}

Samples from myocardial and lung tissues were evaluated for the quantification of hydroxyproline (Hyp) based on the alkaline hydrolysis method. After weighing, samples with $10 \mathrm{mg}$ were detected using the hydroxyproline kit (Nanjing Jiancheng Bioengineering Institute, Nanjing, China). The absorbance of samples was measured spectrophotometrically at wavelength of $550 \mathrm{~nm}$ following the manufacturer's guidelines. The Hyp ( $\mu \mathrm{g} / \mathrm{mg}$ wet weight) concentration was determined from the standard curve.

\section{Statistical Analysis}

The statistical analysis was performed using the SPSS 19.0 software (SPSS, Chicago, IL, United States). Results were expressed as mean \pm SD. Value of $p<0.05$ was considered statistically significant. Normal distribution was assessed using the Kolmogorov-Smirnov test, and homoscedasticity was examined using the Levene's test. All data were found to obey normal distribution and homoscedasticity. Because of the existed difference of body weights among the three groups, data of the HW, LVW, and RVW were compared using analysis of covariance (ANCOVA) with the body mass as a covariate. In additions to the heart and ventricular weights, comparisons of all the other data between each two groups were performed using one-way ANOVA followed by Bonferroni's post hoc analysis. A power analysis was performed to obtain the minimum sample number to ensure the statistical significance among the three groups using the G*Power software. Using a one-way ANOVA with $\alpha=0.05$ and power $=0.8$, we found that the least animal number in each group to establish significance was 4 . Thus, six rats per group for the cardiac muscle histological analysis and myocardial cytokine expression analysis and 12 rats per group for the other tests in the present study were sufficient to secure a good statistical significance.

\section{RESULTS}

\section{Body Mass, Food and Water Intake, Skin Perfusion, and Locomotor Activity}

As shown in Figures $\mathbf{2 A - C}$, no significant difference in body weights, food intake, and water intake was observed before the experiment $(p>0.05)$. Body weights, food intake, and water intake of rats in the $\mathrm{HAH}$ and $\mathrm{HAHO}$ groups were significantly lower than those in the NC group at Weeks 1-4 post hypobaric hypoxia exposure $(p<0.05)$. However, body weights, food intake, and water intake between the HAH group and HAHO group showed no significant difference at any time point $(p>0.05)$. The HAH group exhibited significant increase in the skin blood flux as compared with the NC group (Figure 2D, $p<0.001$ ), while the HAHO group showed significantly lower skin blood flux than the HAH group $(p<0.001)$. No significant difference was found in the horizontal activity among the three groups (Figure 2E, $p>0.05$ ). Moreover, no statistical significance was observed in the vertical activity of rats between the $\mathrm{HAH}$ group and $\mathrm{NC}$ group (Figure 2F, $p>0.05)$. However, the HAHO group showed significant increase in the vertical activity as compared with the NC group and the HAH group $(p<0.05)$.

\section{Hematological Analysis}

According to the hematological results of $\mathrm{RBC}$ indices, the $\mathrm{HAH}$ group had significantly higher $\mathrm{RBC}(p<0.05), \mathrm{Hb}$ $(p<0.001)$, Hct $(p<0.001), \operatorname{MCV}(p<0.001)$, and $\mathrm{MCH}$ $(p<0.001)$ than the NC group (Figures 3A-E). Oxygen enrichment resulted in remarkable decrease in $\mathrm{RBC}(p<0.05)$, 
A

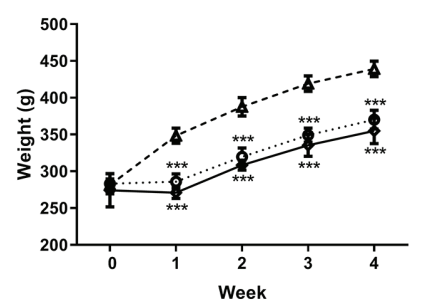

D

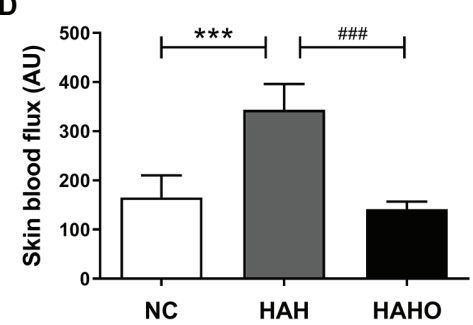

B

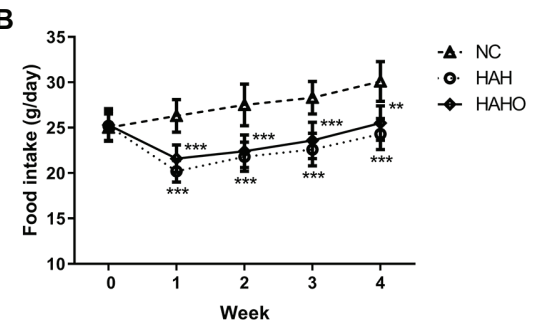

C

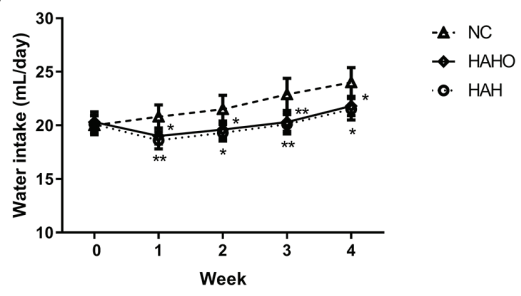

E

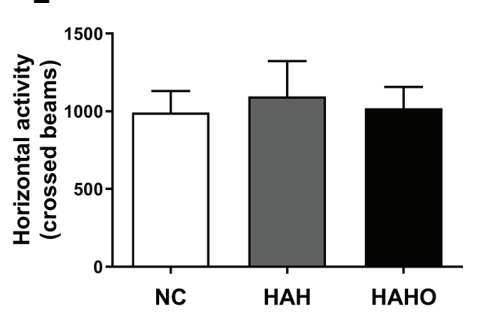

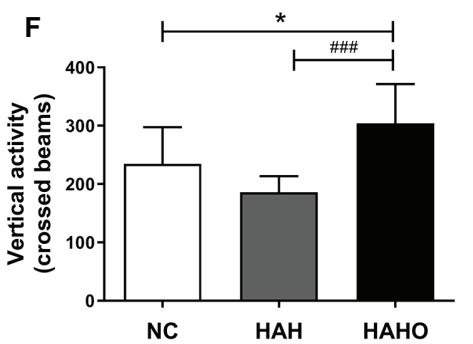

FIGURE 2 | Effects of local oxygen enrichment on (A) body weight, (B) food intake, (C) water intake, (D) skin perfusion, and (E,F) locomotor activity of rats exposed to 4-week hypobaric hypoxia. All data are expressed as mean $\pm \mathrm{SD}(n=12)$. NC, normal control group; $\mathrm{HAH}$, high-altitude hypoxia group; and HAHO, $\mathrm{HAH}$ with oxygen enrichment group. ${ }^{\star} p<0.05$ vs. the NC group, ${ }^{\star \star} p<0.01$ vs. the NC group, ${ }^{\star \star \star} p<0.001$ vs. the NC group, and ${ }^{\# \# \# ~} p<0.001$ vs. the HAH group.

A

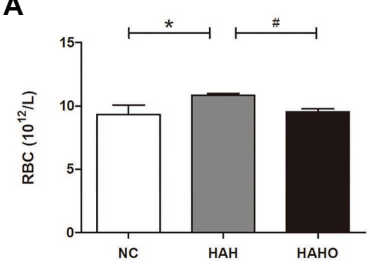

D

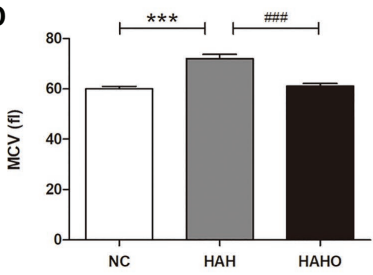

B

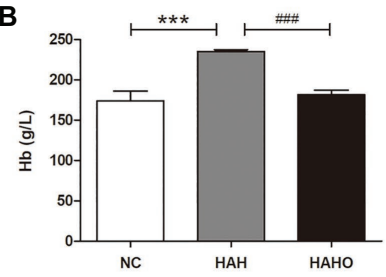

E

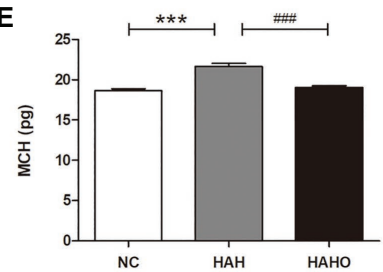

C

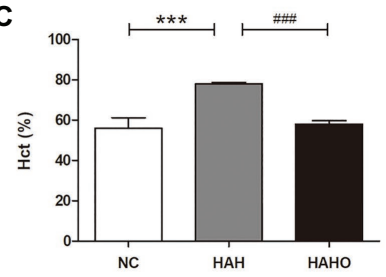

$\mathbf{F}$

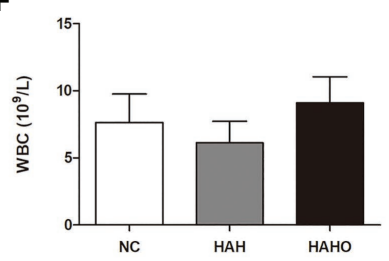

FIGURE 3 | Effects of local oxygen enrichment on hematological parameters of rats exposed to 4-week hypobaric hypoxia, including (A) red blood cell (RBC), (B) hemoglobin (Hb), (C) hematocrit (Hct), (D) mean corpuscular volume (MCV), (E) mean corpuscular hemoglobin (MCH), and (F) white blood cell (WBC). All data are expressed as mean $\pm \mathrm{SD}(n=12)$. NC, normal control group; $\mathrm{HAH}$, high-altitude hypoxia group; and $\mathrm{HAHO}$, high-altitude hypoxia with oxygen enrichment group. ${ }^{\star} p<0.05$ vs. the NC group, ${ }^{\star \star \star} p<0.001$ vs. the NC group, ${ }^{\#} p<0.05$ vs. the HAH group, and ${ }^{\# \# \# ~} p<0.001$ vs. the HAH group.

$\mathrm{Hb}(p<0.001)$, Hct $(p<0.001), \mathrm{MCV}(p<0.001)$, and $\mathrm{MCH}$ $(p<0.001)$ in HAH-exposed rats. Furthermore, no significant change in these erythrocyte indices (RBC, Hb, Hct, MCV, and $\mathrm{MCH})$ was found between the HAHO group and the $\mathrm{NC}$ group $(p>0.05)$. Moreover, no significant difference was observed in WBC among the three groups (Figure 3F, $p>0.05$ ).

\section{Hemodynamic and Ventricular Weight Assessment}

The representative images of hemodynamic results (Figures 4A,B) demonstrated that the levels of RVSP and mPAP in the $\mathrm{HAH}$ group were significantly increased as compared with the NC group (Figures 4 C,D,$p<0.001$ ). The RVSP and mPAP levels of rats in the HAHO group were significantly lower than those in the HAH group $(p<0.05)$. The HAHO group showed significantly higher RVSP than the NC group $(p<0.05)$. The $\mathrm{HAH}$ group exhibited significantly higher RVW $(p<0.01)$ and $\mathrm{RV} /(\mathrm{LV}+\mathrm{S} ; p<0.001)$ than the NC group (Figures $4 \mathbf{G}, \mathbf{H})$, indicating that RV hypertrophy occurred in rats exposed to hypoxia. In comparison with the HAH group, the HAHO group showed significantly lower HW $(p<0.05)$, RVW $(p<0.01)$, and $\mathrm{RV} /(\mathrm{LV}+\mathrm{S} ; p<0.01)$. The HAHO group showed significant 
A

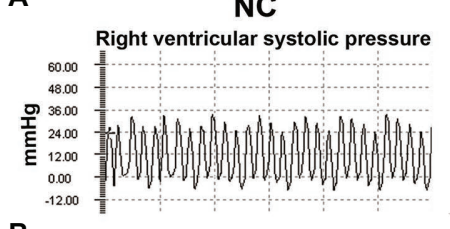

B

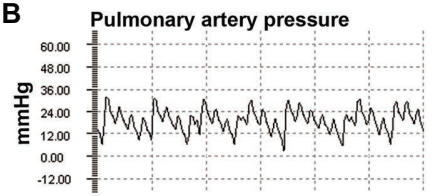

C

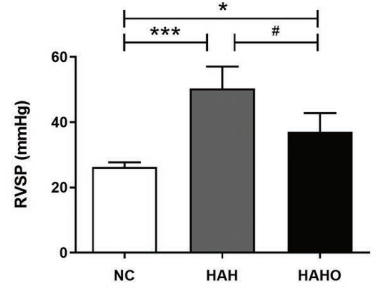

F

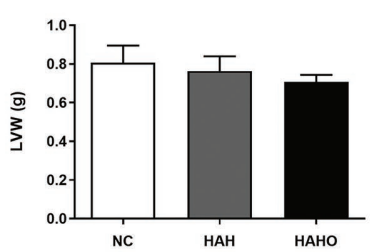

HAH
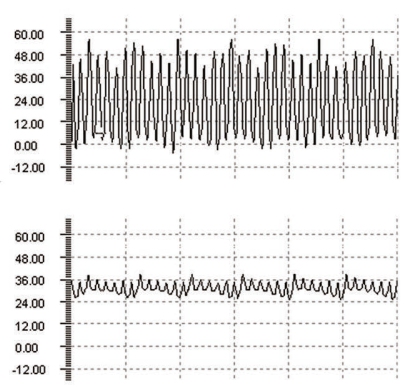

D

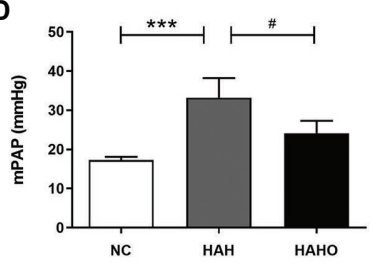

G

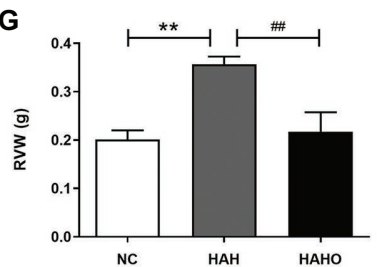

HAHO
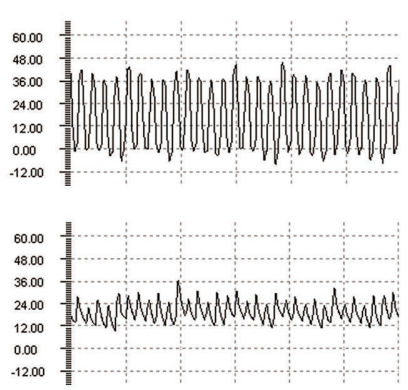

E

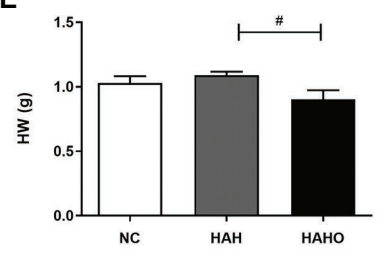

H

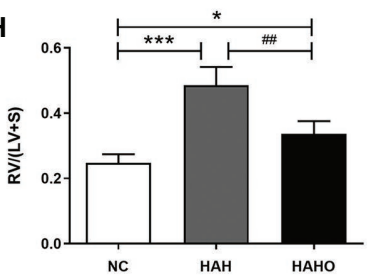

FIGURE 4 | Effects of local oxygen enrichment on hemodynamic indices and ventricular weight of rats exposed to 4-week hypobaric hypoxia. (A,B) Representative images of right ventricular systolic pressure (RVSP) and mean pulmonary artery pressure (mPAP). (C,D) Statistical results of hemodynamic parameters, including RVSP and mPAP. (E-H) Statistical results of ventricular weight, including the heart weight (HW), left ventricular weight (LVW), right ventricular weight (RVW), and the $\mathrm{RV}$ weight to LV plus septum weight ratio [RV/(LV + S)]. All data are expressed as mean $\pm \mathrm{SD}(n=12)$. NC, normal control group; HAH, high-altitude hypoxia group; and $\mathrm{HAHO}$, high-altitude hypoxia with oxygen enrichment group. ${ }^{*} p<0.05$ vs. the NC group, ${ }^{* \star} p<0.05$ vs. the NC group, ${ }^{* \star *} p<0.001$ vs. the NC group, ${ }^{*} p<0.05$ vs. the HAH group, and ${ }^{\# \#} p<0.01$ vs. the HAH group.

increase in $\mathrm{RV} /(\mathrm{LV}+\mathrm{S} ; \mathrm{p}<0.05)$, whereas no significant difference was found in HW or RVW between the HAHO group and the NC group (Figures $4 \mathrm{E}-\mathbf{H}, p>0.05$ ). No significant difference in LVW was detected among the three groups (Figure 4F, $p>0.05$ ).

\section{Cardiac Muscle Histology}

The H\&E staining of cardiac muscle (Figure 5A) demonstrated that 4-week hypobaric hypoxia exerted no significant effect on the LV, as evidenced by no statistically significant difference in the cross-sectional area of LV cardiomyocytes between the $\mathrm{NC}$ group and the HAH group (Figure 5B, $p>0.05$ ). However, the cross section of the RV myocardium showed observable cardiomyocyte enlargement in the HAH group as compared with the NC group (Figure 5A), which was confirmed by the significant increase in the RV cardiomyocyte cross-sectional area (Figure 5C, $p<0.001$ ). No significant difference was found in the cross-sectional area of cardiomyocytes in the LV between the HAHO group and the HAH group $(p>0.05)$. The HAHO group showed significant decrease in the crosssectional area of RV cardiomyocytes as compared with the $\mathrm{HAH}$ group $(p<0.001)$. In addition, no significant change was observed in the cross-sectional area of RV cardiomyocytes between the HAHO group and the NC group $(p>0.05)$.

\section{Myocardial Cytokine Expression}

The HAH group exhibited significantly higher messenger RNA (mRNA) levels of HIF-1 $\alpha$, ACE, and ACE2 in the LV than the NC group (Figures 6A-C, $p<0.001$ ). However, no significant difference was observed in the ACE/ACE2 mRNA ratio between the $\mathrm{HAH}$ group and the $\mathrm{NC}$ group (Figure $6 \mathrm{D}, p>0.05$ ). The HAHO group had significantly lower mRNA expression of HIF- $1 \alpha, \mathrm{ACE}$, and ACE/ACE2 ratio $(p<0.001)$ and higher ACE2 expression $(p<0.001)$ in the LV than the HAH group. In comparison with the $\mathrm{NC}$ group, the HAHO group showed significantly increased gene expression of HIF- $1 \alpha$ and ACE2, and decreased ACE/ACE2 mRNA ratio in the LV $(p<0.001)$. Nevertheless, no significant difference was observed in Col1 $\alpha 1$ or Col3 $\alpha 1$ gene expression in the LV among the three groups (Figures 6E,F, $p>0.05$ ). The HAH group showed significantly higher mRNA levels of HIF-1 $\alpha$, ACE, ACE/ACE2 ratio, Col1 $\alpha 1$, and Col3 $\alpha 1$ in the RV than the NC group (Figures $6 \mathrm{~A}-\mathrm{F}$, $p<0.001)$. The HAHO group exhibited significant decrease in HIF-1 $\alpha$, ACE, ACE/ACE2 ratio, Col1 $\alpha 1$, and Col3 $\alpha 1$ gene 
A
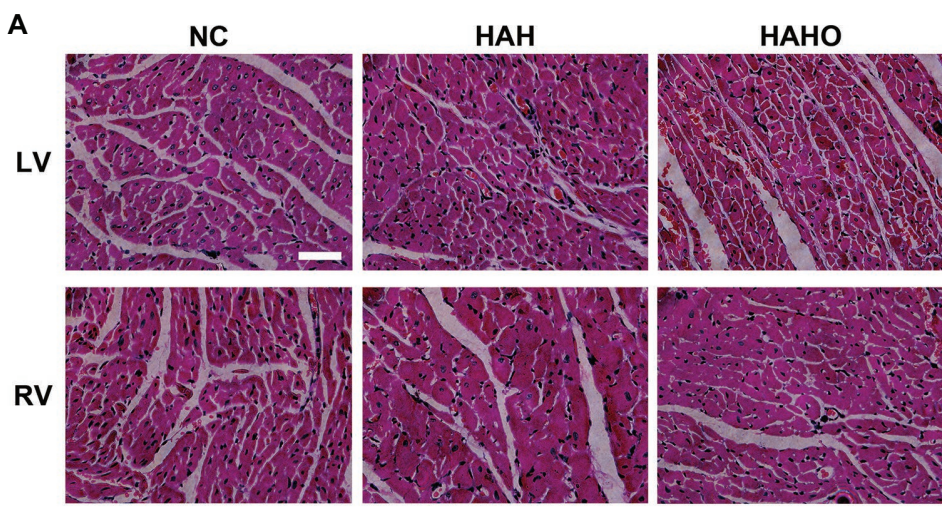

B

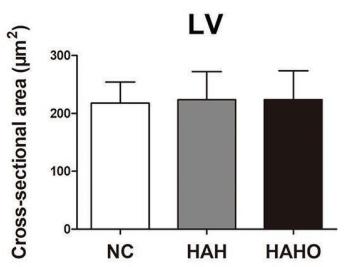

C

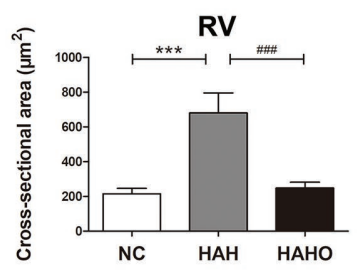

FIGURE 5 | Effects of local oxygen enrichment on the cardiomyocytes of rats exposed to 4-week hypobaric hypoxia according to the histological analysis. (A) H\&E staining images of the cross-sections of the left ventricle (LV) and right ventricle (RV). Scale bars $=50 \mu \mathrm{m}$. (B,C) Statistical results of the cross-sectional area for the LV and RV cardiomyocytes. All data are expressed as mean $\pm \mathrm{SD}(n=6)$. NC, normal control group; HAH, high-altitude hypoxia group; and HAHO, high-altitude hypoxia with oxygen enrichment group. ${ }^{\star \star \star} p<0.001$ vs. the NC group, ${ }^{\# \# ~} p<0.001$ vs. the HAH group.

expression in the RV as compared with the $\mathrm{HAH}$ group (Figures 6A-F, $p<0.001$ ). The mRNA levels of HIF-1 $\alpha$, ACE, $\mathrm{ACE} / \mathrm{ACE} 2$ ratio, Col $1 \alpha 1$, and $\mathrm{Col} 3 \alpha 1$ in the RV of the HAHO group were significantly higher than those in the NC group $(p<0.001)$. However, no difference was found in the ACE2 gene expression in the RV among the three groups (Figure $6 \mathrm{C}$, $p>0.05)$.

Based on the alkaline hydrolysis method, no significant difference was observed in the hydroxyproline content in the LV among the three groups (Figure 6G, $p>0.05$ ). However, the hydroxyproline concentration in the RV of the $\mathrm{HAH}$ group was significantly higher than that in the NC group $(p<0.001)$. Oxygen enrichment significantly decreased the hydroxyproline content in the RV of the HAHO group as compared with the HAH group $(p<0.001)$. The HAHO group exhibited significantly higher hydroxyproline content than that in the NC group $(p<0.05)$. The ELISA results showed no significant change in the cardiac Ang II level in the LV (Figure $\mathbf{6 H}$, $p>0.05$ ) between the NC group and the HAH group, whereas the $\mathrm{HAH}$ group showed a significant increase in myocardial Ang II concentration in the RV as compared with the $\mathrm{NC}$ group $(p<0.001)$. The HAHO group exhibited significantly lower Ang II in the LV $(p<0.001)$ and RV $(p<0.05)$ as compared with the HAH group. The HAHO group showed significant decrease in cardiac Ang II concentration in the LV $(p<0.001)$, and significantly increased Ang II level in the RV $(p<0.01)$.

The Western blotting results (Figures 6I,J) suggested that the protein levels of HIF- $1 \alpha$, ACE, and ACE2 in the LV of the $\mathrm{HAH}$ group were significantly higher than those in the
NC group (Figures $6 \mathbf{K}-\mathbf{M}, p<0.05$ ). The HAH group exhibited no significant difference in the ACE/ACE2 protein ratio as compared with the NC group (Figure $6 \mathbf{N}, p>0.05$ ). The HAHO group showed significantly decreased HIF-1 $\alpha$, ACE, and ACE/ACE2 ratio $(p<0.05)$, and increased ACE2 protein expression $(p<0.001)$ in the LV as compared with the HAH group. The HAHO group exhibited significantly higher protein level of ACE2 $(p<0.001)$ and significantly lower ACE/ACE2 protein ratio $(p<0.05)$ than the $\mathrm{NC}$ group, whereas no significant difference was observed in the HIF-1 $\alpha$ or ACE protein expression between the $\mathrm{HAHO}$ group and the $\mathrm{NC}$ group $(p>0.05)$. HAH resulted in a significant increase in the HIF- $1 \alpha(p<0.001)$ and ACE protein expression $(p<0.01)$ and significantly increased ACE/ACE2 protein ratio $(p<0.05)$, whereas HAH had no effect on the ACE2 protein expression in the RV $(p>0.05)$ of the HAH group as compared with the NC group. Although the HAHO group exhibited significant decrease in the protein levels of HIF- $1 \alpha$, ACE, and ACE/ACE2 ratio $(p<0.05)$, no significant difference in the ACE2 protein expression $(p>0.05)$ was observed in the RV as compared with the HAH group. The HAHO group showed significantly higher protein expression of HIF- $1 \alpha(p<0.01)$ and ACE $(p<0.05)$ than the NC group, whereas no significant difference was found in the protein expression of ACE2 or ACE/ACE2 between the HAHO group and the NC group.

\section{Lung Histology}

According to the $\mathrm{H} \& \mathrm{E}$ staining images (Figure 7A), the $\mathrm{HAH}$ group exhibited increased medial wall thickness of pulmonary arterioles and stenosis as compared with the NC group. 


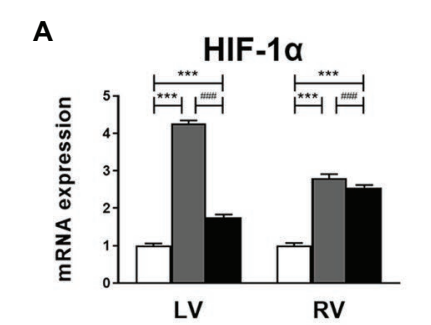

E

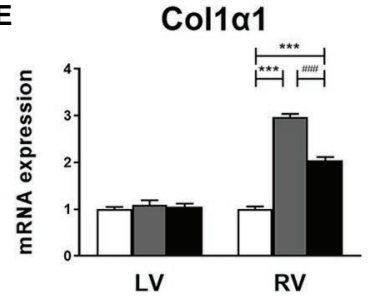

I

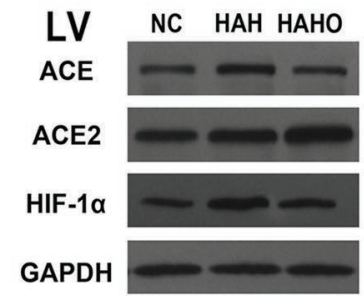

L
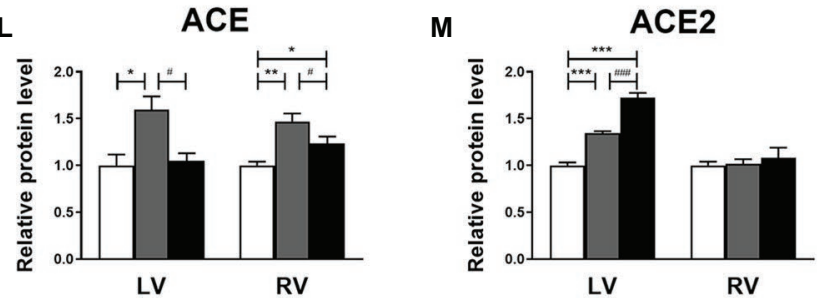

B

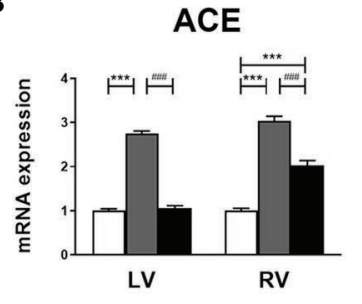

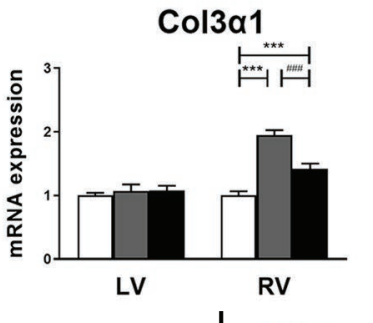

$J$
C

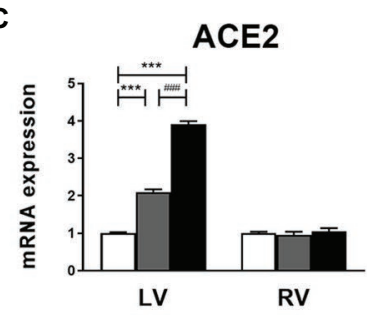

G

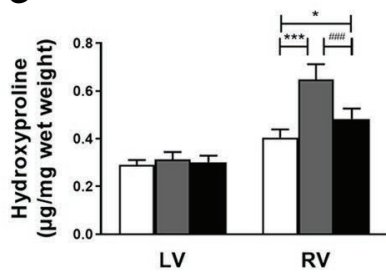

NC HAH HAHO

$198 \mathrm{kDa}$

$92 \mathrm{kDa}$

$90 \mathrm{kDa}$

$36 \mathrm{kDa}$
D

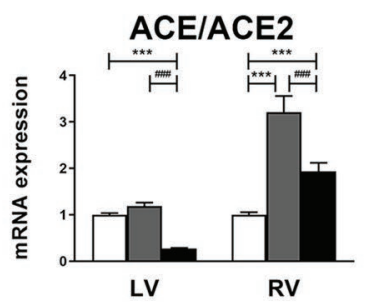

H

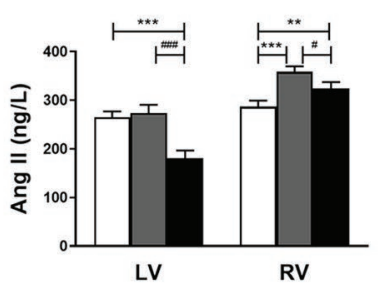

K

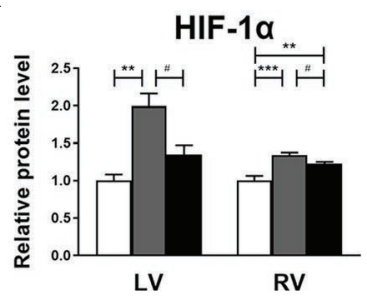

N

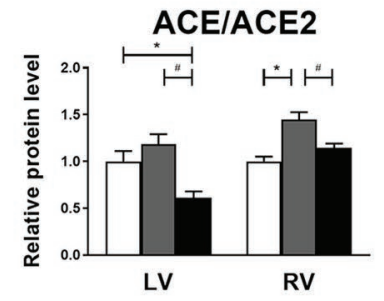

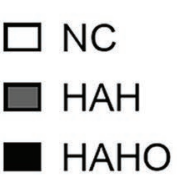

FIGURE 6 | Effects of local oxygen enrichment on the gene expression and protein production of critical cytokines in the LV and RV of rats exposed to 4-week hypobaric hypoxia. (A-F) Statistical results for the mRNA expression of HIF-1 $\alpha$, ACE, ACE2, ACE/ACE2, Col1 $\alpha 1$, and Col3 $\alpha 1$ based on the real-time PCR assays. (G) Quantification of myocardial hydroxyproline concentration based on the alkaline hydrolysis method. (H) Evaluation of myocardial angiotensin II (Ang II) based on ELISA. (I,J) Representative western blotting images for the protein expression of HIF-1 $\alpha$, ACE, ACE2, and GAPDH in the LV and RV. (K-N) Statistical results for the relative protein levels of HIF-1 $\alpha, \mathrm{ACE}, \mathrm{ACE} 2$, and ACE/ACE2 in the LV and RV. All data are expressed as mean $\pm \mathrm{SD}(n=6)$. NC, normal control group; HAH, highaltitude hypoxia group; and $\mathrm{HAHO}$, high-altitude hypoxia with oxygen enrichment group. ${ }^{\star} p<0.05$ vs. the NC group, ${ }^{\star \star} p<0.01$ vs. the $\mathrm{NC}$ group, ${ }^{\star \star \star} p<0.001$ vs. the NC group, ${ }^{\#} p<0.05$ vs. the HAH group, and ${ }^{\# \# \# ~} p 0.001$ vs. the HAH group.

In addition, collagen deposition in the small pulmonary arteries was greater in the HAH group than the NC group (Figure 7B). Statistical analysis showed that the HAH group exhibited significantly increased MWT and WA as compared with the NC group (Figures 7C,D, $p<0.05$ ). Moreover, the HAH group showed significantly higher hydroxyproline concentration in lung tissues than the NC group (Figure 7E, $p<0.001$ ). These changes in the lung histology were partially prevented by oxygen enrichment. In comparison with the HAH group, the HAHO group demonstrated significant decrease in MWT $(p<0.001)$ and WA $(p<0.05)$. Although significant decrease in the hydroxyproline concentration was observed in the HAHO group as compared with the HAH group $(p<0.05)$, the HAHO group showed significantly higher hydroxyproline concentration in lung tissues than the NC group $(p<0.01)$.

\section{Intrapulmonary Cytokine Expression}

The lung mRNA levels of the Col1 $\alpha 1$, Col3 $\alpha 1$, and ET- 1 in the HAH group were significantly higher than those in the NC group (Figures $\mathbf{8 A}-\mathbf{C}, p<0.001$ ). Moreover, the HAH group showed significant increase in the ET-1 pulmonary concentration as compared with the NC group (Figure $8 \mathrm{D}$, $p<0.001)$. Oxygen enrichment inhibited these negative changes in lung tissues, as evidenced by significantly decreased gene expression of Col1 $\alpha 1(p<0.001)$, Col3 $\alpha 1(p<0.001)$, and ET-1 $(p<0.001)$, and reduced ET-1 protein products in the HAHO group $(p<0.01)$ as compared with the HAH group. However, the mRNA levels of Col $1 \alpha 1, \operatorname{Col} 3 \alpha 1$, and ET-1, and the protein concentration of ET-1 in lung tissues were significantly higher than those in the NC group $(p<0.001)$. 
A

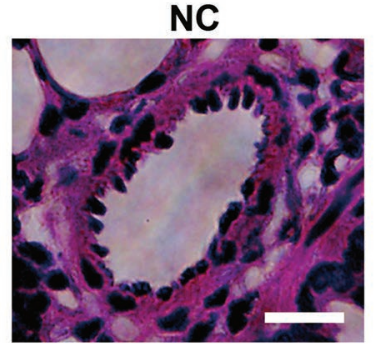

B

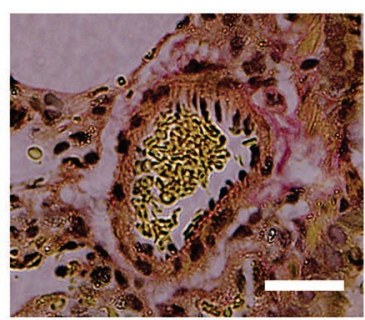

C

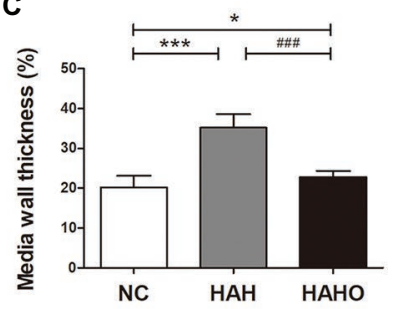

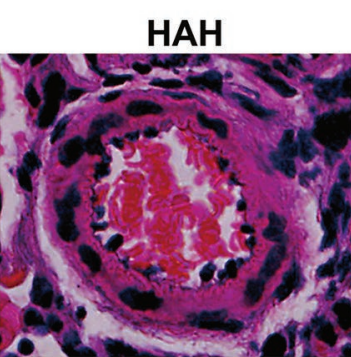

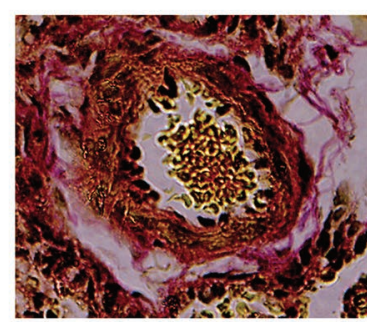

D

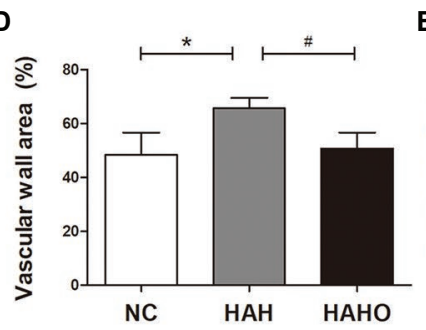

HAHO
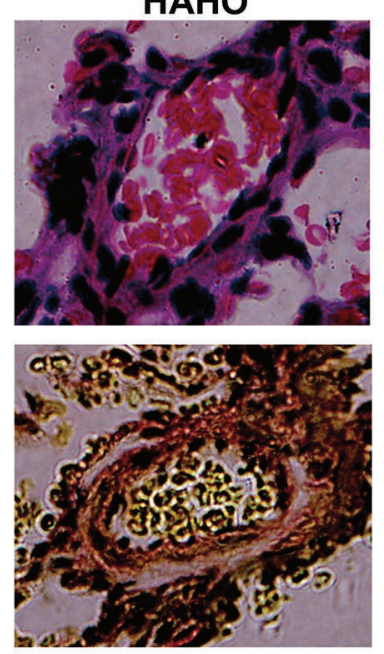

E

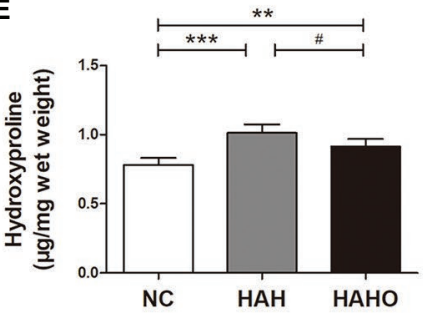

FIGURE 7 | Effects of local oxygen enrichment on the pulmonary arterioles of rats exposed to 4-week hypobaric hypoxia. (A,B) Representative H\&E and Van Gieson (VG) staining images of pulmonary arterioles. Scale bars $=20 \mu \mathrm{m}$. (C,D) Statistical results of the percentage of medial wall thickness (MWT\%) and percentage of vascular wall area (WA\%). MWT\% was calculated as [external diameter (ED) - internal diameter (ID)]/external diameter $\times 100 \%$. WA\% was calculated as [total vascular area (TA) - vascular lumen area (VA)]/TA × 100\%. (E) Quantification of hydroxyproline concentration in lung tissues based on the alkaline hydrolysis method. All data are expressed as mean $\pm \mathrm{SD}(n=12)$. NC, normal control group; $\mathrm{HAH}$, high-altitude hypoxia group; and $\mathrm{HAHO}$, high-altitude hypoxia with oxygen enrichment group. ${ }^{\star} p<0.05$ vs. the NC group, ${ }^{\star *} p<0.01$ vs. the NC group, ${ }^{\star \star \star} p<0.001$ vs. the NC group, ${ }^{\#} p<0.05$ vs. the HAH group, and ${ }^{\# \# \# ~} p<0.001$ vs. the HAH group.

\section{DISCUSSION}

The current available studies for the effects of oxygen enrichment on altitude sickness are mainly clinical investigations, in which suppressed heart rate and diastolic arterial pressure, and increased arterial oxygen saturation resulted from oxygen enrichment were observed for workers at high altitudes (Mcelroy et al., 2000; Li and Liu, 2014; Moraga et al., 2014, 2018). Our group has developed a novel oxygenation device based on the oxygen enrichment membrane technique, and we found that this novel device can induce higher saturation of peripheral oxygen, lower heart rate, and better physical work capacity (Shen et al., 2013). However, to our knowledge, no study has systematically investigated the effects of oxygen enrichment on the injuries of cardiorespiratory structure and function induced by chronic $\mathrm{HAH}$. Based on systematic hematological, histomorphological, and molecular assessments, our results revealed that oxygen enrichment reversed the changes in erythrocyte parameters, largely preserved the cardiac structure and function, and significantly prevented the abnormalities in pulmonary arterioles (including pulmonary vascular remodeling and vasoconstriction) in rats exposed to chronic HAH.
Substantial evidence demonstrates that chronic HAH leads to the body weight reduction in humans or animals (Lippl et al., 2010). Similarly, we observed a significant reduction in the body weight, food, and water intake of rats exposed to chronic HAH, which may result from accelerated metabolic rate and impaired appetite (Tschöp and Morrison, 2001). However, oxygen enrichment for $8 \mathrm{~h}$ per day exerted no obvious effect on $\mathrm{HAH}$-induced decrease in the body mass, food, and water consumption. The increased oxygen supply might not sufficiently compensate for the alteration of digestive metabolism at high altitudes. Moreover, we found that HAH augmented cutaneous microvascular blood flow, which was consistent with previous studies (Carey et al., 2019). Interestingly, oxygen enrichment reversed the increase in the skin blood flow caused by chronic $\mathrm{HAH}$, probably because the increased blood oxygen level was sufficient to compensate for the oxygen consumption without producing microvascular adaptive changes under hypoxic conditions. The open field test has proven to be a useful technique to assess locomotion, exploration, and anxiety of animals (Walsh and Cummins, 1976; Carola et al., 2002; Tatem et al., 2014). Our results demonstrate that chronic HAH induced changes 
A

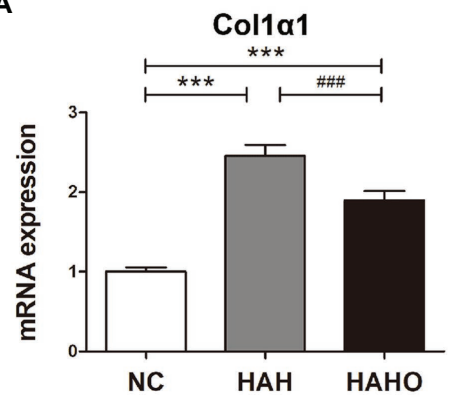

C

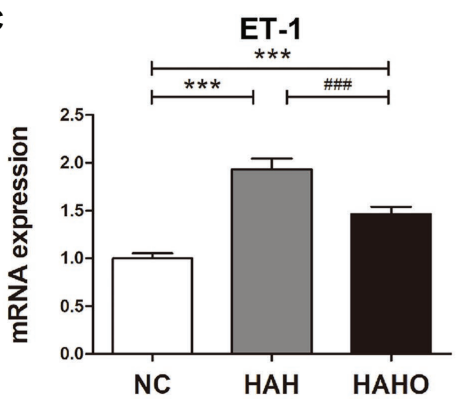

B

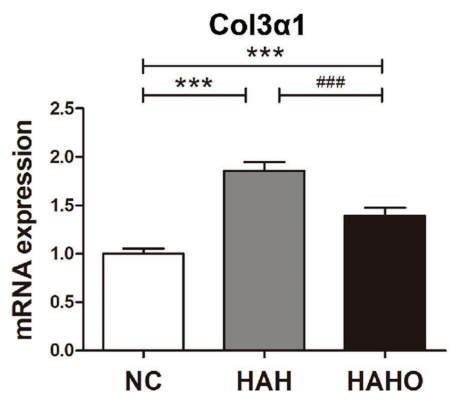

D

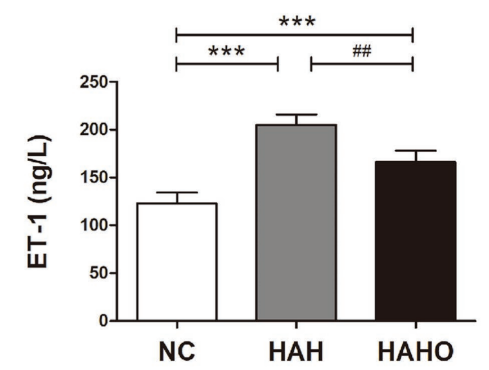

FIGURE 8 | Effects of local oxygen enrichment on the gene expression and protein production of cytokines in lung tissues of rats exposed to 4-week hypobaric hypoxia. (A-C) Statistical results for the mRNA expression of Col1 $\alpha 1$, Col3 $\alpha 1$, and endothelin-1 (ET-1) based on the real-time PCR analysis. (D) Evaluation of the ET-1 protein production in lung tissues based on ELISA. All data are expressed as mean $\pm \mathrm{SD}(n=12)$. NC, normal control group; HAH, high-altitude hypoxia group; and $\mathrm{HAHO}$, high-altitude hypoxia with oxygen enrichment group. ${ }^{\star \star *} p<0.001$ vs. the NC group, ${ }^{\# \#} p<0.01$ vs. the HAH group, and ${ }^{\# \# \#} p<0.001$ vs. the HAH group.

in neither horizontal nor vertical movements, whereas oxygen enrichment greatly increased the locomotor activity, revealing the potential beneficial effects of oxygen enrichment on behavioral responses in chronic HAH rats (Robinson et al., 2018).

It is well known that remarkable alterations occur in hematology to accommodate the HAH condition (Berglund, 1992; Huang et al., 2017). Chronic HAH-induced excessive erythrocytosis increases the blood viscosity, leading to the increased risk of various cardiovascular disorders (e.g., hypertension, vascular thrombosis, and myocardial infarction; Lowe et al., 1997; Tremblay et al., 2019). Our hematological indices revealed that chronic $\mathrm{HAH}$ for 4 weeks increased the amounts of overall RBCs and hemoglobin as well as the volume percentage of RBCs in the blood. Moreover, HAH also increased the average $\mathrm{RBC}$ size and the amount of hemoglobin per RBC, as evidenced by augmented $\mathrm{MCV}$ and $\mathrm{MCH}$. However, $\mathrm{HAH}$ exerted no obvious effect on the number of WBCs. More importantly, our results demonstrated that oxygen enrichment significantly inhibited the increase in the production and volume of erythrocytes and expression of hemoglobin, and almost restored all these parameters to control levels. These findings reveal that oxygen enrichment is able to reverse the changes in erythrocyte parameters induced by chronic $\mathrm{HAH}$, which is potentially beneficial for the decrease in the blood viscosity and subsequent decline in blood pressure (Letcher et al., 1981; Fowkes et al., 1993; Stauffer et al., 2020).

Vascular remodeling and vasoconstriction are common pathological changes under chronic $\mathrm{HAH}$ conditions, resulting in the development of high-altitude pulmonary hypertension (Sylvester et al., 2012; Wilkins et al., 2015). We found that chronic HAH induced thicker medial smooth muscles and severer stenosis of pulmonary arterioles, revealing pathological vascular remodeling in $\mathrm{HAH}$ rats. Our findings also showed that $\mathrm{HAH}$ greatly increased pulmonary mRNA expression and protein products of ET-1, which is a powerful vasoconstrictor involved in hypoxia-induced pulmonary vascular remodeling and pulmonary hypertension (Jankowich et al., 2016; Kylhammar and Rådegran, 2017). We also found that collagen fibers and expression levels of Col $1 \alpha 1, \operatorname{Col} 3 \alpha 1$, and hydroxyproline were significantly increased after chronic $\mathrm{HAH}$, revealing the increased collagen synthesis and development of pulmonary fibrosis in lung tissues (Bernard, 2018; Li and Wu, 2018). Moreover, we observed that medial pulmonary thickness and expression of ET-1 in rat lung tissues were decreased after oxygen enrichment exposure, revealing that $\mathrm{HAH}$-induced pulmonary vasoconstriction and pulmonary vascular remodeling were inhibited. Oxygen enrichment also mitigated HAH-mediated pulmonary fibrosis, as evidenced by decreased expression of Col $1 \alpha 1, \operatorname{Col} 3 \alpha 1$, and hydroxyproline in lung tissues. Previous studies have reported that oxygen enrichment remarkably improves the pulmonary function of subjects exposed to $\mathrm{HAH}$, as evidenced by decreased breaths per minute and reduced incidence of sleep apneas (Luks et al., 1998; Li and Liu, 2014). Our current findings provide further evidence that oxygen enrichment is able to effectively resist chronic $\mathrm{HAH}$-induced negative changes in pulmonary morphology and key molecular expression. 
Pulmonary vasoconstriction and increased pulmonary vascular resistance induce adverse effects on the structure and function of RV under long-term HAH exposure (Wang and Chesler, 2013). Our hemodynamic measurements demonstrated that chronic HAH elevated pulmonary artery pressure and RV systolic pressure in rats, which was in line with previous studies (Naeije and Vanderpool, 2013; Boos et al., 2016; DunhamSnary et al., 2017). It has been shown that the excessive hemodynamic burden imposed on RV leads to subsequent hypertrophic response (Van Der Bruggen et al., 2017; De Man et al., 2019). Our results demonstrated that 4-week HAH exposure remarkably increased the RV weight, Fulton index (an index for RV hypertrophy), and degree of RV cardiomyocyte hypertrophy in rats, whereas LV exhibited no similar changes after HAH exposure. Consistent with our results, $\mathrm{HAH}$-associated $\mathrm{RV}$ dysfunction was also reported in previous clinical and experimental studies (Heath et al., 1973; Kurdziel et al., 2017). Intriguingly, oxygen enrichment partially prevented chronic $\mathrm{HAH}$-induced increase in pulmonary artery pressure and RV systolic pressure, revealing potential protection against pulmonary arterial hypertension. Furthermore, HAH-induced RV remodeling in rats was partially inhibited by oxygen enrichment, characterized by decreased RV weight and reduced cardiomyocyte size. Previous studies only reveal the suppression of heart rate and diastolic arterial pressure of people at high altitudes following oxygen enrichment exposure (Shen et al., 2013; Li and Liu, 2014; Moraga et al., 2018). Our findings further demonstrate that oxygen enrichment has the capability of mitigating the detrimental effects of long-term HAH on cardiac morphology and contractile function in rats.

Substantial studies have confirmed the critical role of hypoxiainducible factors (HIFs) as key regulators responding to oxygen deficiency (Schofield and Ratcliffe, 2004; Majmundar et al., 2010). In the present study, the mRNA and protein expression of HIF-1 $\alpha$ (an oxygen-sensitive subunit) in both the LV and RV was significantly increased after chronic HAH exposure. The mRNA levels of Coll $\alpha 1$ and Col3 $\alpha 1$ and hydroxyproline concentration in the RV were also greatly upregulated under chronic HAH, whereas no similar change occurred in the LV, revealing that hypoxia increased collagen content and promoted myocardial fibrosis in the RV (Watson et al., 2014). Moreover, the expression of ACE and ACE2 in the LV was both upregulated by $\mathrm{HAH}$, and this balance between ACE and ACE2 (the ACE/ ACE2 ratio) maintained the Ang II content (a potent vasoconstrictor). However, the disturbance of the physiological balance between ACE and ACE2 in the RV induced by HAH consequently increased the level of Ang II, which may result in the RV myocyte hypertrophy and cardiac fibrosis (Kim et al., 1995; Schnee and Hsueh, 2000). Oxygen enrichment efficiently mitigated $\mathrm{HAH}$-induced overexpression of HIF-1 $\alpha$ in the LV and RV, and also decreased the Col1 $\alpha 1$ and Col3 $\alpha 1$ expression and cardiac hydroxyproline content in the RV. Furthermore, oxygen enrichment decreased the ACE expression and increased the ACE2 expression in the LV, and thus resulted in the decreased ACE/ACE2 ratio and subsequent decline in Ang II. Although no alteration in ACE2 expression in the RV was observed, the ACE expression and Ang II concentration were decreased following oxygen enrichment exposure. Thus, our findings reveal that oxygen enrichment has the capacity of protecting the LV myocardium from potential damage and also ameliorating the myocardial fibrosis and remodeling in the $\mathrm{RV}$ in response to long-term hypobaric hypoxia in rats. Considering the key role of HIFs in oxygen sensing as intracellular transcriptional regulators, we believe that HIFs might act as a metabolic switch mediating the adaptation of cardiomyocytes to oxygen changes, and thus regulate subsequent ACE/ACE2modulated myocardial remodeling and cardiac fibrosis. The exact HIF-mediated mechanism by which oxygen enrichment improves cardiac function will be identified using gene silencing and overexpressing techniques both in vitro and in vivo in the succeeding studies.

There are several limitations in this research. During the experiment, it is necessary to change the bedding for the animals and replenish the food and water daily in the hypobaric chamber. Because it is impossible for chamber cleaning and food placement without opening the chamber based on the currently available commercial hypobaric device, short-term exposure ( $2 \mathrm{~h} /$ day in this study) to the normoxic environment for animals seems inevitable. Substantial previous studies have also used the similar experiment protocol with the hypobaric chamber exposed to normoxic environment for daily feeding and cleaning (Pichiule et al., 1996; Titus et al., 2007; Xie et al., 2015). In addition, performing the skin perfusion and behavior open field testing for animals under normoxic conditions is another limitation of this study. Similarly, most of previous animal studies investigating the effects of hypobaric hypoxia on the behaviors or blood perfusion were also performed under normoxic conditions due to various technology challenges for direct testing in the hypobaric hypoxia chamber (Takuwa et al., 2013; Kanekar et al., 2015). Moreover, the study was performed by simulating the condition of HAH in the hypobaric hypoxia chamber in the city of Xi'an. It would be interesting to test the effects of oxygen enrichment on HAH-induced cardiorespiratory dysfunction by simulating the heights at different cities in addition to Xi'an in the future studies, which would allow a more transversal extrapolation of the data.

In conclusion, this study demonstrates that oxygen enrichment can effectively inhibit the augmented skin blood flow and improve the locomotor activity in chronic $\mathrm{HAH}$ rats. Oxygen enrichment also has the capability of modifying chronic $\mathrm{HAH}$-induced increased production of erythrocytes. Moreover, oxygen enrichment can attenuate $\mathrm{HAH}$-associated hemodynamic changes and right ventricular hypertrophy in rats. Pulmonary remodeling and vasoconstriction were also significantly inhibited following oxygen enrichment. This study provides the first evidence revealing that the oxygen enrichment technique has the capability of resisting chronic $\mathrm{HAH}$-induced cardiopulmonary dysfunction in animals, and offers preliminary rationale for future clinical trials focusing on evaluating whether oxygen enrichment mitigates chronic HAH-induced cardiopulmonary damage for workers and residents on the plateau. The oxygen enrichment technique can not only be designed for individual use, but more importantly can be designed for providing diffusion oxygen supply in local 
space (e.g., room, tent, and vehicle) to decrease the equivalent altitude and attenuate chronic mountain sickness.

\section{DATA AVAILABILITY STATEMENT}

The original contributions presented in the study are included in the article/supplementary material, further inquiries can be directed to the corresponding authors.

\section{ETHICS STATEMENT}

The animal study was reviewed and approved by Institutional Animal Care and Use Committee of the Fourth Military Medical University.

\section{REFERENCES}

Adde, F. V., Alvarez, A. E., Barbisan, B. N., and Guimarães, B. R. (2013). Recommendations for long-term home oxygen therapy in children and adolescents. J. Pediatr. 89, 6-17. doi: 10.1016/j.jped.2013.02.003

Azad, P., Stobdan, T., Zhou, D., Hartley, I., Akbari, A., Bafna, V., et al. (2017). High-altitude adaptation in humans: from genomics to integrative physiology. J. Mol. Med. 95, 1269-1282. doi: 10.1007/s00109-017-1584-7

Bärtsch, P. (1992). Treatment of high altitude diseases without drugs. Int. J. Sports Med. 13, S71-S74. doi: 10.1055/s-2007-1024600

Berglund, B. (1992). High-altitude training. Aspects of haematological adaptation. Sports Med. 14, 289-303. doi: 10.2165/00007256-199214050-00002

Bernard, K. (2018). Collagen biosynthesis in pulmonary fibrosis: unraveling the metabolic web. Am. J. Respir. Cell Mol. Biol. 58, 545-546. doi: 10.1165/rcmb.2017-0350ED

Bolton, C. E., Annandale, J. A., and Ebden, P. (2006). Comparison of an oxygen concentrator and wall oxygen in the assessment of patients undergoing long term oxygen therapy assessment. Chron. Respir. Dis. 3, 49-51. doi: 10.1191/1479972306cd098oa

Boos, C. J., O’hara, J. P., Mellor, A., Hodkinson, P. D., Tsakirides, C., Reeve, N., et al. (2016). A four-way comparison of cardiac function with normobaric normoxia, normobaric hypoxia, hypobaric hypoxia and genuine high altitude. PLoS One 11:e0152868. doi: 10.1371/journal.pone.0152868

Boussuges, A., Molenat, F., Burnet, H., Cauchy, E., Gardette, B., Sainty, J. M., et al. (2000). Operation Everest III (Comex '97): modifications of cardiac function secondary to altitude-induced hypoxia. An echocardiographic and Doppler study. Am. J. Respir. Crit. Care Med. 161, 264-270. doi: 10.1164/ajrccm.161.1.9902096

Carey, D., Thanaj, M., Davies, T., Gilbert-Kawai, E., Mitchell, K., Levett, D. Z. H., et al. (2019). Enhanced flow-motion complexity of skin microvascular perfusion in Sherpas and lowlanders during ascent to high altitude. Sci. Rep. 9:14391. doi: 10.1038/s41598-019-50774-0

Carola, V., Dolimpio, F., Brunamonti, E., Mangia, F., and Renzi, P. (2002). Evaluation of the elevated plus-maze and open-field tests for the assessment of anxiety-related behaviour in inbred mice. Behav. Brain Res. 134, 49-57. doi: 10.1016/S0166-4328(01)00452-1

Davis, C., and Hackett, P. (2017). Advances in the prevention and treatment of high altitude illness. Emerg. Med. Clin. North Am. 35, 241-260. doi: 10.1016/j.emc.2017.01.002

De Man, F. S., Handoko, M. L., and Vonk-Noordegraaf, A. (2019). The unknown pathophysiological relevance of right ventricular hypertrophy in pulmonary arterial hypertension. Eur. Respir. J. 53:1900255. doi: 10.1183/13993003.00255-2019

Deneke, S. M., and Fanburg, B. L. (1980). Normobaric oxygen toxicity of the lung. N. Engl. J. Med. 303, 76-86. doi: 10.1056/NEJM198007103030204

Dunham-Snary, K. J., Wu, D., Sykes, E. A., Thakrar, A., Parlow, L. R. G., Mewburn, J. D., et al. (2017). Hypoxic pulmonary vasoconstriction: from molecular mechanisms to medicine. Chest 151, 181-192. doi: 10.1016/j.chest.2016.09.001

Fowkes, F. G., Lowe, G. D., Rumley, A., Lennie, S. E., Smith, F. B., and Donnan, P. T. (1993). The relationship between blood viscosity and blood

\section{AUTHOR CONTRIBUTIONS}

DJ, CT, and EL designed the research. XS, XD, JC, and ZY performed the experiments. XS, XD, JC, ZY, KX, DJ, and EL analyzed data. XS, JC, and DJ wrote the paper. All authors have read and approved the manuscript.

\section{FUNDING}

We acknowledge the support from Shaanxi Youth Science and Technology New Star Project (No. 2019KJXX-087), the Research Project of Shaanxi Provincial Department of Education (No. 19JK0242), and the Shaanxi Provincial Natural Science Foundation of China (No. 2020SF-084).

pressure in a random sample of the population aged 55 to 74 years. Eur. Heart J. 14, 597-601. doi: 10.1093/eurheartj/14.5.597

Hackett, P. H., and Roach, R. C. (2001). High-altitude illness. N. Engl. J. Med. 345, 107-114. doi: 10.1056/NEJM200107123450206

Heath, D., Edwards, C., Winson, M., and Smith, P. (1973). Effects on the right ventricle, pulmonary vasculature, and carotid bodies of the rat of exposure to, and recovery from, simulated high altitude. Thorax 28, 24-28. doi: 10.1136/thx.28.1.24

Heyboer, M. 3rd, Sharma, D., Santiago, W., and Mcculloch, N. (2017). Hyperbaric oxygen therapy: side effects defined and quantified. Adv. Wound Care 6, 210-224. doi: 10.1089/wound.2016.0718

Huang, H., Liu, B., Wu, G., Xu, G., Sun, B. D., and Gao, Y. Q. (2017). Hematological risk factors for high-altitude headache in Chinese men following acute exposure at 3,700 m. Front. Physiol. 8:801. doi: 10.3389/fphys.2017.00801

Jankowich, M. D., Wu, W. C., and Choudhary, G. (2016). Association of elevated plasma endothelin-1 levels with pulmonary hypertension, mortality, and heart failure in African American individuals: the Jackson heart study. JAMA Cardiol. 1, 461-469. doi: 10.1001/jamacardio.2016.0962

Kanekar, S., Bogdanova, O. V., Olson, P. R., Sung, Y. H., D'anci, K. E., and Renshaw, P. F. (2015). Hypobaric hypoxia induces depression-like behavior in female Sprague-dawley rats, but not in males. High Alt. Med. Biol. 16, 52-60. doi: 10.1089/ham.2014.1070

Kasic, J. F., Yaron, M., Nicholas, R. A., Lickteig, J. A., and Roach, R. (1991). Treatment of acute mountain sickness: hyperbaric versus oxygen therapy. Ann. Emerg. Med. 20, 1109-1112. doi: 10.1016/S0196-0644(05)81385-X

Kim, S., Ohta, K., Hamaguchi, A., Yukimura, T., Miura, K., and Iwao, H. (1995). Angiotensin II induces cardiac phenotypic modulation and remodeling in vivo in rats. Hypertension 25, 1252-1259. doi: 10.1161/01.HYP.25.6.1252

Kurdziel, M., Wasilewski, J., Gierszewska, K., Kazik, A., Pytel, G., Wacławski, J., et al. (2017). Echocardiographic assessment of right ventricle dimensions and function after exposure to extreme altitude: is an expedition to $8000 \mathrm{~m}$ hazardous for right ventricular function? High Alt. Med. Biol. 18, 330-337. doi: 10.1089/ham.2017.0019

Kylhammar, D., and Rådegran, G. (2017). The principal pathways involved in the in vivo modulation of hypoxic pulmonary vasoconstriction, pulmonary arterial remodelling and pulmonary hypertension. Acta Physiol. 219, 728-756. doi: 10.1111/apha.12749

León-Velarde, F., and Richalet, J. P. (2006). Respiratory control in residents at high altitude: physiology and pathophysiology. High Alt. Med. Biol. 7, 125-137. doi: 10.1089/ham.2006.7.125

León-Velarde, F., Villafuerte, F. C., and Richalet, J. P. (2010). Chronic mountain sickness and the heart. Prog. Cardiovasc. Dis. 52, 540-549. doi: 10.1016/j.pcad.2010.02.012

Letcher, R. L., Chien, S., Pickering, T. G., Sealey, J. E., and Laragh, J. H. (1981). Direct relationship between blood pressure and blood viscosity in normal and hypertensive subjects. Role of fibrinogen and concentration. Am. J. Med. 70, 1195-1202. doi: 10.1016/0002-9343(81)90827-5

Li, Y., and Liu, Y. (2014). Oxygen enrichment and its application to life support systems for workers in high-altitude areas. Int. J. Occup. Environ. Health 20, 207-214. doi: 10.1179/2049396714Y.0000000068 
Li, P., and Wu, G. (2018). Roles of dietary glycine, proline, and hydroxyproline in collagen synthesis and animal growth. Amino Acids 50, 29-38. doi: 10.1007/ s00726-017-2490-6

Lippl, F. J., Neubauer, S., Schipfer, S., Lichter, N., Tufman, A., Otto, B., et al. (2010). Hypobaric hypoxia causes body weight reduction in obese subjects. Obesity (Silver Spring) 18, 675-681. doi: 10.1038/oby.2009.509

Lowe, G. D., Lee, A. J., Rumley, A., Price, J. F., and Fowkes, F. G. (1997). Blood viscosity and risk of cardiovascular events: the Edinburgh artery study. Br. J. Haematol. 96, 168-173. doi: 10.1046/j.1365-2141.1997.8532481.x

Luks, A. M., Van Melick, H., Batarse, R. R., Powell, F. L., Grant, I., and West, J. B. (1998). Room oxygen enrichment improves sleep and subsequent day-time performance at high altitude. Respir. Physiol. 113, 247-258. doi: 10.1016/S0034-5687(98)00068-1

Majmundar, A. J., Wong, W. J., and Simon, M. C. (2010). Hypoxia-inducible factors and the response to hypoxic stress. Mol. Cell 40, 294-309. doi: 10.1016/j.molcel.2010.09.022

Mcelroy, M. K., Gerard, A., Powell, F. L., Prisk, G. K., Sentse, N., Holverda, S., et al. (2000). Nocturnal O2 enrichment of room air at high altitude increases daytime $\mathrm{O} 2$ saturation without changing control of ventilation. High Alt. Med. Biol. 1, 197-206. doi: 10.1089/15270290050144190

Moore, L. G. (2001). Human genetic adaptation to high altitude. High Alt. Med. Biol. 2, 257-279. doi: 10.1089/152702901750265341

Moraga, F. A., Jiménez, D., Richalet, J. P., Vargas, M., and Osorio, J. (2014). Periodic breathing and oxygen supplementation in Chilean miners at high altitude (4200m). Respir. Physiol. Neurobiol. 203, 109-115. doi: 10.1016/j. resp.2014.09.001

Moraga, F. A., López, I., Morales, A., Soza, D., and Noack, J. (2018). The effect of oxygen enrichment on cardiorespiratory and neuropsychological responses in workers with chronic intermittent exposure to high altitude (ALMA, 5,050 m). Front. Physiol. 9:187. doi: 10.3389/fphys.2018.00187

Murray, A. J., Montgomery, H. E., Feelisch, M., Grocott, M. P. W., and Martin, D. S. (2018). Metabolic adjustment to high-altitude hypoxia: from genetic signals to physiological implications. Biochem. Soc. Trans. 46, 599-607. doi: 10.1042/BST20170502

Naeije, R., and Vanderpool, R. (2013). Pulmonary hypertension and chronic mountain sickness. High Alt. Med. Biol. 14, 117-125. doi: 10.1089/ham.2012.1124

Nagatomo, F., Fujino, H., Kondo, H., and Ishihara, A. (2012). Oxygen concentrationdependent oxidative stress levels in rats. Oxidative Med. Cell. Longev. 2012:381763. doi: $10.1155 / 2012 / 381763$

Nasilowski, J., Przybylowski, T., Zielinski, J., and Chazan, R. (2008). Comparing supplementary oxygen benefits from a portable oxygen concentrator and a liquid oxygen portable device during a walk test in COPD patients on longterm oxygen therapy. Respir. Med. 102, 1021-1025. doi: 10.1016/j.rmed.2008.02.005

Peacock, A. J. (1998). ABC of oxygen: oxygen at high altitude. BMJ 317, 1063-1066. doi: 10.1136/bmj.317.7165.1063

Penaloza, D., and Arias-Stella, J. (2007). The heart and pulmonary circulation at high altitudes: healthy highlanders and chronic mountain sickness. Circulation 115, 1132-1146. doi: 10.1161/CIRCULATIONAHA.106.624544

Pichiule, P., Chávez, J. C., Boero, J., and Arregui, A. (1996). Chronic hypoxia induces modification of the N-methyl-D-aspartate receptor in rat brain. Neurosci. Lett. 218, 83-86. doi: 10.1016/S0304-3940(96)13113-X

Robinson, L., Spruijt, B., and Riedel, G. (2018). Between and within laboratory reliability of mouse behaviour recorded in home-cage and open-field. J. Neurosci. Methods 300, 10-19. doi: 10.1016/j.jneumeth.2017.11.019

Schnee, J. M., and Hsueh, W. A. (2000). Angiotensin II, adhesion, and cardiac fibrosis. Cardiovasc. Res. 46, 264-268. doi: 10.1016/S0008-6363(00)00044-4

Schofield, C. J., and Ratcliffe, P. J. (2004). Oxygen sensing by HIF hydroxylases. Nat. Rev. Mol. Cell Biol. 5, 343-354. doi: 10.1038/nrm1366

Shen, G., Wu, X., Tang, C., Yan, Y., Liu, J., Guo, W., et al. (2013). An oxygen enrichment device for lowlanders ascending to high altitude. Biomed. Eng. Online 12:100. doi: 10.1186/1475-925x-12-100

Stauffer, E., Loyrion, E., Hancco, I., Waltz, X., Ulliel-Roche, M., Oberholzer, L., et al. (2020). Blood viscosity and its determinants in the highest city in the world. J. Physiol. 598, 4121-4130. doi: 10.1113/JP279694

Sylvester, J. T., Shimoda, L. A., Aaronson, P. I., and Ward, J. P. (2012). Hypoxic pulmonary vasoconstriction. Physiol. Rev. 92, 367-520. doi: 10.1152/ physrev.00041.2010

Takuwa, H., Masamoto, K., Yamazaki, K., Kawaguchi, H., Ikoma, Y., Tajima, Y., et al. (2013). Long-term adaptation of cerebral hemodynamic response to somatosensory stimulation during chronic hypoxia in awake mice. J. Cereb. Blood Flow Metab. 33, 774-779. doi: 10.1038/jcbfm.2013.16

Tatem, K. S., Quinn, J. L., Phadke, A., Yu, Q., Gordish-Dressman, H., and Nagaraju, K. (2014). Behavioral and locomotor measurements using an open field activity monitoring system for skeletal muscle diseases. J. Vis. Exp. 29:51785. doi: $10.3791 / 51785$

Titus, A., Rao, B. S., Harsha, H., Ramkumar, K., Srikumar, B., Singh, S., et al. (2007). Hypobaric hypoxia-induced dendritic atrophy of hippocampal neurons is associated with cognitive impairment in adult rats. Neuroscience 145, 265-278. doi: 10.1016/j.neuroscience.2006.11.037

Tremblay, J. C., Hoiland, R. L., Howe, C. A., Coombs, G. B., Vizcardo-Galindo, G. A., Figueroa-Mujíca, R. J., et al. (2019). Global REACH 2018: high blood viscosity and hemoglobin concentration contribute to reduced flow-mediated dilation in high-altitude excessive erythrocytosis. Hypertension 73, 1327-1335. doi: 10.1161/HYPERTENSIONAHA.119.12780

Tschöp, M., and Morrison, K. M. (2001). Weight loss at high altitude. Adv. Exp. Med. Biol. 502, 237-247. doi: 10.1007/978-1-4757-3401-0_16

Van Der Bruggen, C. E. E., Tedford, R. J., Handoko, M. L., Van Der Velden, J., and De Man, F. S. (2017). RV pressure overload: from hypertrophy to failure. Cardiovasc. Res. 113, 1423-1432. doi: 10.1093/cvr/cvx145

Vargas, E., and Spielvogel, H. (2006). Chronic mountain sickness, optimal hemoglobin, and heart disease. High Alt. Med. Biol. 7, 138-149. doi: 10.1089/ ham.2006.7.138

Vonk Noordegraaf, A., Westerhof, B. E., and Westerhof, N. (2017). The relationship between the right ventricle and its load in pulmonary hypertension. J. Am. Coll. Cardiol. 69, 236-243. doi: 10.1016/j.jacc.2016.10.047

Walsh, R. N., and Cummins, R. A. (1976). The open-field test: a critical review. Psychol. Bull. 83, 482-504. doi: 10.1037/0033-2909.83.3.482

Wang, Z., and Chesler, N. C. (2013). Pulmonary vascular mechanics: important contributors to the increased right ventricular afterload of pulmonary hypertension. Exp. Physiol. 98, 1267-1273. doi: 10.1113/expphysiol.2012.069096

Watson, C. J., Collier, P., Tea, I., Neary, R., Watson, J. A., Robinson, C., et al. (2014). Hypoxia-induced epigenetic modifications are associated with cardiac tissue fibrosis and the development of a myofibroblast-like phenotype. Hum. Mol. Genet. 23, 2176-2188. doi: 10.1093/hmg/ddt614

West, J. B. (2002). Commuting to high altitude: value of oxygen enrichment of room air. High Alt. Med. Biol. 3, 223-235. doi: 10.1089/15270290260131948

West, J. B. (2003). Improving oxygenation at high altitude: acclimatization and O2 enrichment. High Alt. Med. Biol. 4, 389-398. doi: 10.1089/152702903769192340

West, J. B. (2012). High-altitude medicine. Am. J. Respir. Crit. Care Med. 186, 1229-1237. doi: $10.1164 / \mathrm{rccm} .201207-1323 \mathrm{CI}$

West, J. B. (2015). High-altitude medicine. Lancet Respir. Med. 3, 12-13. doi: 10.1016/S2213-2600(14)70238-3

Wilkins, M. R., Ghofrani, H. A., Weissmann, N., Aldashev, A., and Zhao, L. (2015). Pathophysiology and treatment of high-altitude pulmonary vascular disease. Circulation 131, 582-590. doi: 10.1161/CIRCULATIONAHA.114.006977

Xie, X., Wang, G., Zhang, D., Zhang, Y., Zhu, Y., Li, F., et al. (2015). Activation of peroxisome proliferator-activated receptor $\gamma$ ameliorates monocrotalineinduced pulmonary arterial hypertension in rats. Biomed. Rep. 3, 537-542. doi: $10.3892 / b r .2015 .465$

Yang, T., Li, X., Qin, J., Li, S., Yu, J., Zhang, J., et al. (2015). High altitudeinduced borderline pulmonary hypertension impaired cardiorespiratory fitness in healthy young men. Int. J. Cardiol. 181, 382-388. doi: 10.1016/j. ijcard.2014.12.044

Zhu, H. T., Bian, C., Yuan, J. C., Liao, X. J., Liu, W., Zhu, G., et al. (2015). Hyperbaric oxygen therapy ameliorates acute brain injury after porcine intracerebral hemorrhage at high altitude. Crit. Care 19:255. doi: 10.1186/ s13054-015-0976-8

Conflict of Interest: The authors declare that the research was conducted in the absence of any commercial or financial relationships that could be construed as a potential conflict of interest.

Copyright (๑) 2021 Shao, Dong, Cai, Tang, Xie, Yan, Luo and Jing. This is an openaccess article distributed under the terms of the Creative Commons Attribution License (CC BY). The use, distribution or reproduction in other forums is permitted, provided the original author(s) and the copyright owner(s) are credited and that the original publication in this journal is cited, in accordance with accepted academic practice. No use, distribution or reproduction is permitted which does not comply with these terms. 